\title{
The Impact of Edge Deletions on the Number of Errors in Networks
}

\author{
Christian Glacet ${ }^{\star}$, Nicolas Hanusse ${ }^{\star}$, and David Ilcinkas ${ }^{\star}$ \\ LaBRI, University of Bordeaux, CNRS, INRIA
}

\begin{abstract}
In this paper, we deal with an error model in distributed networks. For a target $t$, every node is assumed to give an advice, ie.to point to a neighbour that take closer to the destination. Any node giving a bad advice is called a liar. Starting from a situation without any liar, we study the impact of topology changes on the number of liars.

More precisely, we establish a relationship between the number of liars and the number of distance changes after one edge deletion. Whenever $\ell$ deleted edges are chosen uniformly at random, for any graph with $n$ nodes, $m$ edges and diameter $D$, we prove that the expected number of liars and distance changes is $O\left(\frac{\ell^{2} D n}{m}\right)$ in the resulting graph. The result is tight for $\ell=1$. For some specific topologies, we give more precise bounds.
\end{abstract}

Keywords: dynamic graph, errors and faults, shortest path and routing

\section{Introduction}

\subsection{The Search Problem.}

Everyone has already faced the problem of reaching a destination in an uncertain network. This is typically the case whenever you are in an unknown city, without a map, and you aim at reaching, let us say, the closest cash machine. The only thing you can do is ask for some information from people in the street. Unfortunately, there is no evidence that all the information you get is reliable.

Nowadays, in a communication network, a corresponding situation can occur. Let us consider the routing task. Due to its dynamicity (change of topology, time required to update local information) and its large-scale size, current networks are not immune to faults and crashes. It is no more realistic to blindly trust the data stored locally at each node. For instance, the Border Gate Protocol (BGP) used in Internet to route messages between autonomous systems implicitly assumes that some paths are known to reach any target. Ideally, these paths are as short as possible. Unfortunately, many messages do not reach their destination because no paths are temporally known although some paths could exist. Is there a way to find such paths?

In the following, for a given target $t$, we informally refer to a liar as a node containing bad information about the location of $t$. The word liar is used

\footnotetext{
* This work is granted by the european project EULER.
} 
even if nodes have not necessarily malicious intentions, but are simply ignorant. A series of papers [HKK04,HKKK08,HIKN10] tackle the problem of locating a target (node, resource, data, ...) in presence of liars.

A first model was introduced by Kranakis and Krizanc [KK99]. They designed algorithms for searching in distributed networks having the ring or the torus topology, when a node has a constant probability of being a liar. A more realistic model was proposed by Hanusse et al. [HKK04]: the number of liars is a parameter $k$ and during a routing query, the information stored at every node is unchanged. The main performance measure is the number of edge traversals during a request. Several algorithms, either generic or dedicated to some topologies, and bounds are presented in [HKK04,HKKK08,HIKN10] and are typically of the form $O\left(d+k^{O(1)}\right)$ (for path,grids, expanders,...) or $\Theta\left(d+2^{O(k)}\right)$ for bounded degree graphs, $d$ being the distance between the source and the target.

In these papers, there is an implicit assumption: the number of liars is small. Our goal is to evaluate whether this is realistic or not. Starting from a network without any liar, we aim at estimating bounds on the number of liars obtained after few changes of topology. It turns out that this problem is related to the problem of estimating the number of distance changes after few edge/node deletions or insertions. In this paper, we focus on edge deletions for the following reasons: it is a more atomic event than node deletion (any node deletion can be represented as a sequence of edge deletions) and a deletion is much more dramatic than an insertion in our context. On the one hand, after one deletion, there is potentially no known or existing path toward the target and on the other hand, after one insertion, we could only miss a shortcut.

\subsection{Related Works}

The influence of topology changes on graph parameters is studied in several works. In [CG84,SBvL87], it is proved that for any sequence of $\ell$ edge deletions that do not disconnect the graph, the diameter $D$ of any unweighted graph turns to be less than $D(\ell+1)$. Our work is also related to the computation of the most vital node of a shortest path [NPW03], that is the node whose removal results in the largest increase of the distance for a given pair of source/target, and the Vickrey pricing of edges [HS01].

Recently, some work on dynamic data structures for shortest paths/distance computation problems has been proposed. By dynamic, we mean that the data structures can tolerate some topology changes in a given network. A dynamic network model defines how the underlying graph changes/evolves over time. More precisely, the following type of models are usually considered:

- Evolving models without constraint: it consists in an "online" insertion and/or suppression of links and/or nodes. Roughly speaking, if $G(t)$ is the network at time $t$ then $G(0)$ and $G(t)$ can be quite different.

- Failure model: $G(t)$ is a subgraph of $G(0)$. In practice, we consider that few nodes/links are removed from $G(0)$. 
The most standard model of dynamic network is the following: starting from an initial graph, a sequence of $\ell$ insertions/deletions of edges/nodes is done. Each query has to be answered taking into account the $\ell$ updates. The most naive solution consists in recomputing all shortest paths after any update but it is generally quite costly. For instance, the update time of the fastest dynamic algorithms for the all-pairs shortest path takes $O\left(n^{2}\right.$ polylog $\left.(n)\right)$ [DI04,Tho04]. It turns out that in the failure model, it is not always necessary to recompute all shortest paths. Some solutions provide efficient data structures dedicated to the problem of reporting shortest path or distance queries for $\ell=1$. More precisely, we can distinguish data structures dedicated to exact solution [DTCR08,BK09] or constant approximation of the solution [KB10,CLPR10], that is a constant factor of shortest path/distance after one edge/node deletion. The challenge is to handle efficiently more than $\ell>1$ updates. To our knowledge, the more general result is the $\ell$-sensitivity distance [CLPR10] oracle for which a data structure of size $O\left(\ell s n^{1+1 / s} \log n\right)$ is able to approximate the distance between any node pairs within a factor $O(s \cdot \ell)$ for undirected graphs in $O\left(\ell \log ^{O(1)} n\right)$ time. Note that the data structures report distances / routing paths, given the knowledge of the $\ell$ nodes/edges to avoid. They provide a similar result for weighted graphs and, only if $\ell \leq 2$, for compact routing.

In these works, the implicit model is the one of a strong adversary model: the worst sequence of updates. This is sometimes too pessimistic to explain and to model macroscopic observations done on real dynamic networks. In the following, we will also consider the random fault adversary model: any sequence of $\ell$ updates has the same probability to occur. Estimating the number of distance changes in a dynamic network can be used to get a tight analysis of the update time. In King's algorithm analysis ([Kin99] - section 2.1 or [Ber09]), the update time to maintain a shortest path tree turns to be $O(D$. \#number of distance changes from the root) for connected bounded degree graphs whenever $\ell=1$. Our results allow to analyse the random fault case.

\subsection{Contribution}

Models The network is modelled by a graph $G=(V, E)$ of $|V|=n$ nodes and $|E|=m$ edges. $G$ is assumed to be unweighted and can be disconnected. Note that $D$ correspond to the maximum diameter of all the connected components. The neighbourhood of vertex $u$ is noted $\Gamma(u)$ and includes $u$ itself. Given a target located at a node $t$, each node $u \in V \backslash\{t\}$ has an advice $\operatorname{Adv}(u) \in \Gamma(u) \backslash\{u\}$. Node $u$ is a truthteller if $\operatorname{Adv}(u)$ belongs to a shortest path from $u$ to $t$ and otherwise $u$ is a liar. The set of advice $A$ can also define a directed subgraph of $G$, noted $G_{A}$. There is an $\operatorname{arc}(u, v)$ in $G_{A}$ if and only if $v=\operatorname{Adv}(u)$. Whenever there exists no liar,$G_{A}$ is a shortest path spanning tree rooted at $t$.

We shall investigate two main parameters:

- the number of liars $k=k_{G}(A)$ for a set of advice $A$ in graph $G$

- and the size of the set $\mathcal{S}$ of nodes whose distance to $t$ has changed after one edge deletion. 
For instance, in Figure 2, we have $n-D$ lying nodes pointing toward a dead-end in the rightmost drawing and $D-1$ nodes whose distance to $t$ has changed after one edge deletion.

Given a graph $G$ without any liar and a target $t$, we aim at analysing the combined effect of the choice of set of advice $A$ and the set of $\ell$ edges. Note that $A$ is not arbitrary since we assume that $G$ has no liar. After a deletion, it may happen that the resulting graph turns to be disconnected. Nodes that do not belong to the connected component of node $t$ become liars. The set of advice is unchanged with a potential exception: if a deleted edge was used as an advice, one extremity needs to draw another advice among its current neighbours. We focus on two models:

- The adversary model: this model represents a worst-case analysis. An adversary has the capacity of choosing $A$, the set of edges to remove and the potential new advice to draw. Thus, $k$ is maximal in this model.

- The random fault model: $A$ is assumed to be chosen uniformly at random in the universe of set of advice without liars for the given graph. The set of edges to delete and the potential new advice are chosen uniformly at random.

$\widetilde{G}$ is the resulting subgraph of $G$ after $\ell$ deletions .

Results The majority of our results focus on the random fault model since most of the results in the adversary model are simpler. However, it is interesting to take the two models in order to see a potential gap between them.

More precisely, our main result deals with the random fault model : after $\ell$ deleted edges are chosen uniformly at random, for any graph of $n$ nodes, $m$ edges and diameter $D$, we prove that the expected number of liars, $\mathbb{E}(k)$, and the expected number of distance changes $\mathbb{E}(|\mathcal{S}|)$ is in $O\left(\frac{\ell^{2} D n}{m}\right)$ in the resulting graph.

Table 1 shows our results after one deletion in both models. Note that the notation $\Theta(\cdot)$ simultaneously stands for a lower bound and an upper bound. The lower bound means that there exists a graph of the family for which the number of liars is in $\Omega(\cdot)$.

Note that an edge deletion does not necessarily imply the creation of a liar even if some nodes have changed their distance to $t$, for instance the complete graph ${ }^{1}$ ). Conversely, some liars can appear without any change of distance within the graph.

For the family of graphs of diameter $D$, it is easy to reach the bound for the adversary model : just take a path of $D$ nodes and add a star of $n-D$ leaves to one extremity. If $t$ is located to the other extremity, one edge deletion can

\footnotetext{
${ }^{1}$ In the complete graph, if an edge is removed,

- either this edge was used as an advice by node $u \in V$, in this case $d(u, t)=2$ and any new advice takes closer to $t$;

- or not and therefore no liar is created.
} 
Fig. 1. Number of liars induced by a single edge deletion

\begin{tabular}{l|l|l} 
Topology & Adversary & Random fault \\
\hline Graphs of diameter $D$ & $\Theta(n)$ & $\Theta\left(\frac{D n}{m}\right)$ \\
\hline Square Grid & $\Theta(\sqrt{n})$ & $\Theta(1)$ \\
\hline ErdsRnyi model & $\frac{n-1}{4}+1$ & $\Theta\left(\frac{1}{n}\right)$ \\
\hline Hypercube & $\log n-1$ & $\Theta\left(\frac{1}{\log n}\right)$ \\
\hline
\end{tabular}

disconnect the graph implying $k$ and $|\mathcal{S}|$ to be of linear size. Even if somebody would restrict edge deletion to connected graphs, we can easily claim a lower bound of $\Omega(n-D-1)$ (see Figure 2).

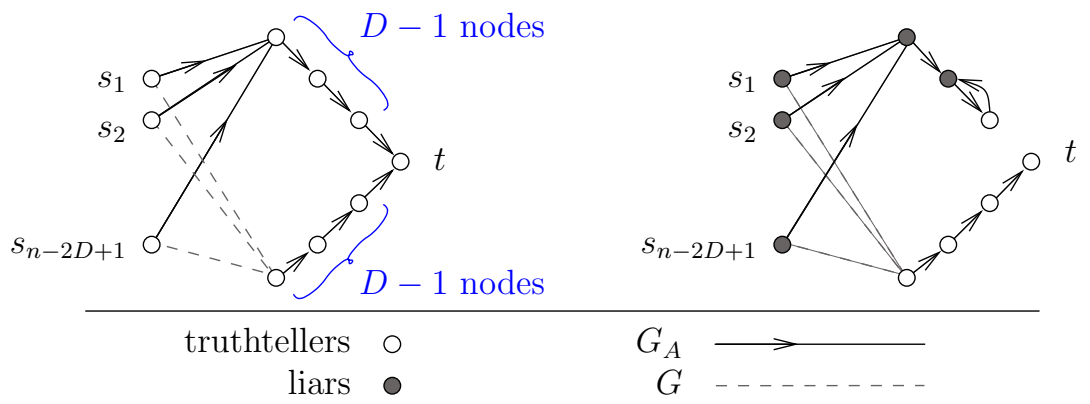

Fig. 2. An example of an edge deletion that creates $n-D-1$ liars

The structure of the paper is the following: we start by exhibiting a relationship between the number of distance changes and the number of liars induced by an arbitrary edge deletion (Lemma 2). Then, we prove that, in the random fault model, $\mathbb{E}(|S|) \leq \frac{D n}{m}$. Combining with Lemma 2, we show that $\mathbb{E}(k)<2 D$ (Theorem 1). This result is then improved (Theorem 2) and generalized to $\ell$ edge deletions (Theorem 4). More precisely, we prove that the deletion of $\ell$ random edges creates at most $O\left(\frac{\ell^{2} n D}{m}\right)$ liars. In the last section, we give more precise bounds for specific topologies (see Table 1). 


\section{General Results}

\subsection{Preliminaries}

We start by presenting some notations and some easy facts used in our paper.

$\widetilde{G}_{e} G$ after deletion of edge $e, \widetilde{G}_{e}=(V, E \backslash e)$, or simply $\widetilde{G}$.

$d(u, v)$ distance in $G$ from $u$ to $v$.

$d_{\widetilde{G}}(u, v)$ distance in $\widetilde{G}$ from $u$ to $v$.

$\Gamma(X) X$ 's neighbourhood in $G, \Gamma(X)=\bigcup_{x \in X} \Gamma(x)$

$\operatorname{Adv}^{-1}(X)$ set of nodes advising another node that belongs to $X, i e \cdot \operatorname{Adv}^{-1}(X)=\{u \in V \mid \operatorname{Adv}(u) \in X\}$

$\mathcal{F}(e)$ indicates if edge $e=\{x, y\}$ belongs to the set of advised edges $G_{A}$.

More precisely, $\mathcal{F}(e)=1$ if $\operatorname{Adv}(x)=y \vee \operatorname{Adv}(y)=x$ and $\mathcal{F}(e)=0$ otherwise.

Many of our proofs are based on the notion of $(s, t)$-arterial edges:

Definition 1. An edge $\{x, y\}$ is $(s, t)$-arterial if it belongs to all shortest paths from $s$ to $t$

The deletion of a $(s, t)$-arterial edge implies

$$
\text { the event } \mathcal{E}_{s, t}: d_{\widetilde{G}}(s, t)>d(s, t)
$$

Otherwise, there exists a shortest path from $s$ to $t$ which does not contain $\{x, y\}$. The set of arterial edges from $s$ to $t$ is denoted $\mathcal{C}_{s, t}$. It follows that

Lemma 1. The distance from $s$ to $t$ is modified by a single edge deletion if and only if this edge belongs to $\mathcal{C}_{s, t}$.

\subsection{Relationships Between the Number of Liars and the Number of Distance Changes}

Let us denote $\mathcal{S}=\mathcal{S}_{t}^{e}=\left\{s \in V \mid\right.$ the deletion of $e$ implies $\left.\mathcal{E}_{s, t}\right\}$ the set of nodes that have changed their distance to $t$ after the deletion of some edge $e$.

Lemma 2. In any graph containing $k_{0}$ liars, the number of liars $k$ after deletion of an edge $e$ always satisfies

$$
\left|A d v^{-1}(\mathcal{S}) \backslash \mathcal{S}\right| \leq k \leq\left|A d v^{-1}(\mathcal{S})\right|+\mathcal{F}(e)+k_{0}
$$

Proof. In any graph with $k_{0}$ liars, after one edge deletion, we study the impact for every node (ie.advice) on the resulting number of liars $k$. For every node $u$ with $v \in V$ and $\operatorname{Adv}(u)=v$, we have :

$$
d_{G}(u, t)-d_{G}(v, t) \in \begin{cases}\{1\} & \text { if } u \text { is a truthteller } \\ \{0,-1\} & \text { if } u \text { is a liar }\end{cases}
$$

If $u \notin \mathcal{S}$ and $v \in \mathcal{S}$ then

$$
d_{\widetilde{G}}(u, t)-d_{\widetilde{G}}(v, t) \in \begin{cases}\{0,-1\} & \text { if } u \text { was a truthteller } \\ \{-1\} & \text { if } u \text { was a liar }\end{cases}
$$


hence $u$ becomes (or remains) a liar. The minimum number of liars after one deletion is then

$$
k \geq\left|\operatorname{Adv}^{-1}(\mathcal{S}) \backslash \mathcal{S}\right|
$$

Let us now consider the upper bound. First assume that the removed edge $e \neq$ $\{u, v\}$. If $v \notin \mathcal{S}$ then $u$ remains a liar:

- $u \in \mathcal{S}$ and $v \notin \mathcal{S}$ then :

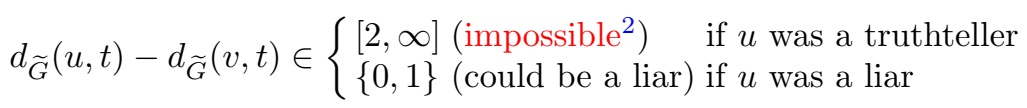

- if $u \notin \mathcal{S}$ and $v \notin \mathcal{S}$ then $d_{\widetilde{G}}(u, t)-d_{\widetilde{G}}(v, t)=d_{G}(u, t)-d_{G}(v, t)$.

If $u \in S$ and $v \in S$, then $d_{\widetilde{G}}(u, t)-d_{\widetilde{G}}(v, t) \in\{1,0,-1\}$, so $u$ could be a liar or not independently of its previous state. So, the maximum number of liars added by one edge deletion is at most $\left|\operatorname{Adv}^{-1}(\mathcal{S})\right|$. Then

$$
k \leq\left|\operatorname{Adv}^{-1}(\mathcal{S})\right|+k_{0}
$$

Finally, if the removed edge $e=\{u, v\}$, ie.F $\mathcal{F}(e)=1$, then $u$ has to change its advice and becomes a liar. In the worst situation, the number of liars is then increased by one.

\subsection{Upper Bounds for $\ell=1$ Deleted Edge in the Random Fault Model}

According to our model, and as we have already seen in Lemma 2, liars apparition is due to distance changes and advice deletion.

\section{Number of distance changes}

Lemma 3. In any m-edge graph $G=(V, E)$, if an edge, chosen uniformly at random, is removed from $E$ then the number $|S|$ of distance changes satisfies

$$
\forall t \in V: \mathbb{E}(|\mathcal{S}|)=\frac{1}{m} \sum_{s \in V \backslash\{t\}}\left|\mathcal{C}_{s, t}\right| .
$$

Proof. From Lemma 1, if edge $\{x, y\}$ is chosen uniformly at random in $E$ then $\forall s \in V:$

$$
\mathbb{P}\left(\mathcal{E}_{s, t}\right)=\frac{\left|\mathcal{C}_{s, t}\right|}{m}
$$

Let $X_{s, t}$ be a random variable defined by $X_{s, t}=1$ if $\mathcal{E}_{s, t}$, and $X_{s, t}=0$ otherwise. We get

$$
\mathbb{E}(|\mathcal{S}|)=\mathbb{E}\left(\sum_{s \in V \backslash\{t\}} X_{s, t}\right)=\sum_{s \in V \backslash\{t\}} \mathbb{E}\left(X_{s, t}\right)=\sum_{s \in V \backslash\{t\}} \mathbb{P}\left(\mathcal{E}_{s, t}\right)=\frac{1}{m} \sum_{s \in V \backslash\{t\}}\left|\mathcal{C}_{s, t}\right|
$$

\footnotetext{
$\overline{2}$ impossible because $u$ and $v$ are neighbours
} 
Corollary 1. For any n-node, m-edge graph of diameter D, after one random edge deletion, we have in the random fault model

$$
\mathbb{E}(|\mathcal{S}|) \leq \frac{D(n-1)}{m} .
$$

Proof. In a graph of diameter $D$, by definition, all shortest paths lengths are at most $D$. So, $\forall s \in V \backslash\{t\}$, there is at most $D(s, t)$-arterial edges in $E$.

Number of Liars Applying Lemma 2, we get

Corollary 2. For any n-node, $m$-edge graph of diameter $D$ and maximal degree $\Delta$ without liar, after one random edge deletion, we have $\mathbb{E}(k) \leq \frac{(D \Delta+1)(n-1)}{m}$.

This turns to be optimal up to a constant factor for bounded degree graphs (see Theorem 3). However, this is not the case whenever the graph has nodes of unbounded degree.

Theorem 1. For graphs of diameter $D$ without liar, after random one edge deletion, we have

$$
\mathbb{E}(k) \leq 2 D
$$

Proof. According to Lemma 2, for any edge $e$, if $\left|\mathcal{S}_{e, t}^{e}\right|$ nodes change their distance to $t$, then the number of added liars after deletion of edge $e$, is at most $\left|\operatorname{Adv}^{-1}(\mathcal{S})\right|+\mathcal{F}(e) \leq \sum_{s \in \mathcal{S}}\left|\operatorname{Adv}^{-1}(s)\right|+\mathcal{F}(e)$.

Take the possible $m$ edge deletions trials and consider the $m$ corresponding sets $\mathcal{S}_{i}$ for $i$ going from 1 to $m$. In a given trial in which event $\mathcal{E}_{s, t}$ occurs, each node $s$ adds at most $\left|\operatorname{Adv}^{-1}(s)\right| \leq \operatorname{degree}(s)-1$ liars (excluding itself) since $G$ contains initially no liar and at least one neighbour of $s$ is closer to $t$ than $s$. Since $\forall s \in V \backslash\{t\}$, event $\mathcal{E}_{s, t}$ can occur in at most $\left|\mathcal{C}_{s, t}\right| \leq D$ instances among the $m$ ones. It follows that for given $s, \sum_{i: s \in \mathcal{S}_{i}}\left|\operatorname{Adv}^{-1}(s)\right| \leq D(\operatorname{degree}(s)-1)$.

Thus, for any $i \in[1, m]$, we have $k_{\widetilde{G}_{i}} \leq\left|\operatorname{Adv}^{-1}\left(\mathcal{S}_{i}\right)\right|+\mathcal{F}(e) \leq \sum_{s \in \mathcal{S}_{i}} \operatorname{degree}(s)$. Summing over all values of $i$, we get

$$
\sum_{i=1}^{m} k_{\widetilde{G}_{i}} \leq \sum_{i=1}^{m} \sum_{s \in \mathcal{S}_{i}} \operatorname{degree}(s)=\sum_{s \in V \backslash\{t\}} \sum_{i: s \in \mathcal{S}_{i}} \operatorname{degree}(s) \leq \sum_{s \in V \backslash\{t\}} D \cdot \operatorname{degree}(s)=2 m \cdot D
$$

It turns out that $\mathbb{E}\left(k_{\widetilde{G}}\right) \leq \frac{2 m D}{m}=2 D$.

A more precise bound can be found by reasoning on a hierarchical cutaway of $G$ from distance 0 to $D$ with respect to target $t$. The following part shows a detailed proof based on this principle to get a tighter upper bound $\left(\leq \frac{D n}{m}\right)$.

Nodes in danger Let $\mathcal{T}_{u, v}$ be the set of nodes that have at least one shortest path to $v \in V$ through $u \in V$. Let $L_{i}=\{x \in V \mid d(x, t)=i\}$ be the set of nodes at distance $i$ from $t$. Every node $v \in \mathcal{T}_{x, t}$ with $x \in L_{i}$ is in danger ${ }^{3}$ with respect to level $i$ if and only if only one shortest path from $x$ to $t$ exists. In Figure 3, all nodes from sets $\mathcal{T}_{x_{2}, t}$ and $\mathcal{T}_{x_{3}, t}$ are in danger.

\footnotetext{
${ }^{3}$ can potentially turns into a liar
} 
Distances and shortest paths Let $\mathcal{C}_{i}=\left\{\{x, y\} \mid x \in L_{i}, y \in L_{i-1} \wedge \Gamma(x) \cap L_{i-1}=\right.$ $\{y\}\}$ be the set of arterial edges between $L_{i}$ and $L_{i-1}$. Let $B_{t}(i-1)$ be the set of nodes at distance at most $i-1$ from $t$. If $G$ is not connected then the set of edges that does not belong to the connected component of $t$ is $\mathcal{C}_{\infty}$.

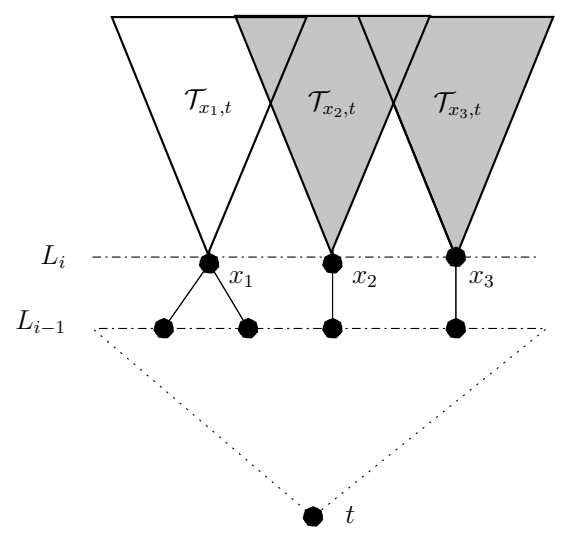

Fig. 3. $G$ levels and nodes in danger (grey filled areas)

Lemma 4. For any graph, containing an arbitrary number of liars $k_{0}$. If the edge $\{x, y\}$ is deleted uniformly at random between levels $L_{i}$ and $L_{i-1}$ and $i \leq D$ then the number of liars added $k_{n e w}{ }^{4}$ is

$$
\mathbb{E}\left(k_{n e w} \mid\{x, y\} \in \mathcal{C}_{i}\right) \leq \frac{n-\left|B_{t}(i-1)\right|}{\left|\mathcal{C}_{i}\right|}
$$

and $\mathbb{E}\left(k_{\text {new }} \mid\{x, y\} \in \mathcal{C}_{\infty}\right)=0$.

Proof. The number of arterial edges between $L_{i}$ and $L_{i-1}$ is

$$
\left|\mathcal{C}_{i}\right|=\left|\left\{x \in L_{i},\left|\Gamma(x) \cap L_{i-1}\right|=1\right\}\right|
$$

Since all the nodes in danger belong to $\bigcup_{x \in L_{i}} \mathcal{T}_{x, t}$, the average number of liars added by a random deletion between levels $L_{i}$ and $L_{i-1}$ is at most

$$
\mathbb{E}\left(k_{n e w} \mid\{x, y\} \in \mathcal{C}_{i}\right) \leq \frac{\left|\bigcup_{x \in L_{i}} \mathcal{T}_{x, t}\right|}{\left|\mathcal{C}_{i}\right|} \leq \frac{n-B_{t}(i-1)}{\left|\mathcal{C}_{i}\right|}
$$

Note that some of the $k_{0}$ liars could belong to $\bigcup_{x \in L_{i}} \mathcal{T}_{x, t}$. These liars will be counted twice.

\footnotetext{
$\overline{ }^{4}$ Note that $k_{\text {new }}=k-k_{0}$
} 
Theorem 2. For $D \geq 2$, the numbers of liars added $k_{\text {new }}$ by deleting an edge chosen uniformly at random in $E$ is

$$
\mathbb{E}\left(k_{\text {new }}\right) \leq \frac{D\left(n-\frac{D-1}{2}\right)}{m} \leq \frac{D(n-1)}{m}
$$

For $D=1, \mathbb{E}\left(k_{\text {new }}\right)=k_{\text {new }}=0$. This result holds for arbitrary graphs, unnecessarily connected.

Proof. The average number of liars added is the sum of the expected number of liars induced by deletions between every levels $L_{1}, L_{2}, \ldots, L_{D}$

$\mathbb{E}\left(k_{\text {new }}\right)=\sum_{i=1}^{\infty}\left(\mathbb{E}\left(k_{\text {new }} \mid\{x, y\} \in \mathcal{C}_{i}\right) \times \mathbb{P}\left(\{x, y\} \in \mathcal{C}_{i}\right)\right)=\sum_{i=1}^{D}\left(\mathbb{E}\left(k_{\text {new }} \mid\{x, y\} \in \mathcal{C}_{i}\right) \times \mathbb{P}\left(\{x, y\} \in \mathcal{C}_{i}\right)\right)$

The probability of deleting an edge at level $i$ is

$$
\mathbb{P}\left(\{x, y\} \in \mathcal{C}_{i}\right)=\frac{\left|\mathcal{C}_{i}\right|}{m}
$$

Thus, from lemma 4

$$
\mathbb{E}\left(k_{\text {new }}\right) \leq \sum_{i=1}^{D} \frac{n-\left|B_{t}(i-1)\right|}{\left|\mathcal{C}_{i}\right|} \times \frac{\left|\mathcal{C}_{i}\right|}{m} \leq \frac{D n}{m}-\frac{1}{m} \sum_{i=1}^{D}\left|B_{t}(i-1)\right|
$$

$\forall i \in D,\left|B_{t}(i-1)\right| \geq i-1$, hence, the average number of liars added is

$$
\mathbb{E}\left(k_{\text {new }}\right) \leq \frac{D n}{m}-\frac{D(D-1)}{2 m} \leq \frac{D\left(n-\frac{D-1}{2}\right)}{m}
$$

\subsection{Lower Bound for $\ell=1$ in the Random Fault Model}

Theorem 3. For any integers $n, m, D$ such that $m \geq n \geq 2 D \geq 20$,

- there exists a graph of $n+O(1)$ nodes, $\Theta(m)$ edges and diameter $D$ for which the expected number of liars after a random edge deletion is greater than $\frac{(D-8) n}{32 m}$.

- there exists a graph of $\Theta(n)$ nodes, $\Theta(m)$ edges and diameter $D$ for which the expected number of distance changes after a random edge deletion is $\Omega\left(\frac{D n}{m}\right)$.

Proof. Let us consider a graph $H$ (see $H_{1}$ in Figure 4) built in the following way: take a complete graph of size $r$ and a stable of size $r^{\prime}$. Add two extra nodes $u, v$ and link them to the $r+r^{\prime}$ nodes. This graph has diameter 2, $r+r^{\prime}+2$ nodes and $\frac{r(r-1)}{2}+2\left(r+r^{\prime}\right)$ edges. Take now four copies of $H$ named $H_{1}, H_{2}$, $H_{3}$ and $H_{4}$. For $i$ going from 1 to 4 , link $u_{i}$ to $v_{(i \bmod 4)+1}$ by a path of $D / 2-4$ 


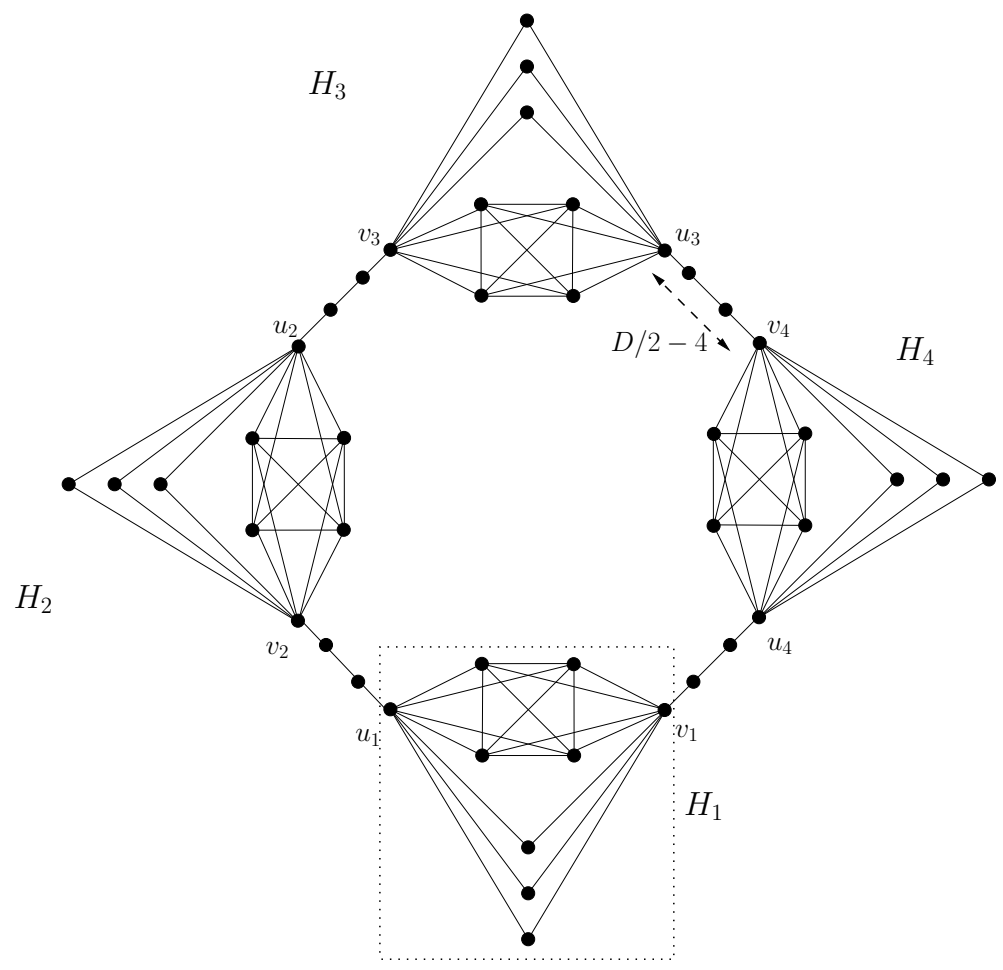

Fig. 4. Sample graph in which the lower bound is reached 
edges. The resulting graph $G$ has diameter $D$. We set up $r=\left\lceil\sqrt{\frac{m-D}{2}}\right\rceil$ and $r^{\prime}=\left\lceil\frac{n-D}{4}-r\right\rceil$. It follows that $G$ has $n+O(1)$ nodes. The total number of edges is $\Theta(n)$. This graph is presented in Figure 4.

Without loss of generality, assume now that target $t$ is either between $u_{1}$ and $v_{2}$ or belongs to $H_{1}$. In the first case, it follows that every node of $H_{3}$ (excluding $v_{3}$ and potentially $u_{3}$ ) has $v_{3}$ as advice toward $t$. The probability that the deleted random edge belongs to the path from $u_{2}$ to $v_{3}$ is $p=\frac{D-8}{2 m}$. The expected number of liars/distance changes is at least $p\left(r+r^{\prime}\right) \geq \frac{(D-8) n}{16 m}$.

For the second case, every node of $H_{3}$ excluding $u_{3}$ or $v_{3}$ can point arbitrarily to $u_{3}$ and $v_{3}$. Take the node given by the majority. If $v_{3}$ (resp. $u_{3}$ ) is chosen, then $p$ corresponds to the probability that the deleted random edge belongs to the path from $u_{2}$ to $v_{3}$ (resp. $v_{3}$ to $u_{4}$ ). The expected number of liars turns to be greater than $p\left(\frac{r+r^{\prime}}{2}\right) \geq \frac{(D-8) n}{32 m}$.

In this last case, in order to get a similar lower bound for the expected number of distance changes, we just have to slightly modify each $H_{i}$ copy. We just substitute each node of the stable set by an edge between two nodes. Each copy turns to have $r+2 r^{\prime}$ nodes and $\frac{r(r-1)}{2}+2 r+3 r^{\prime}$ edges. We only have to consider the distance change from $t$ and $r^{\prime}$ nodes of this new set. To have $r^{\prime}=\Theta(n)$, we might have to consider a graph $G$ with $\Theta(n)$ nodes (at most $2 n$ is enough).

\section{$3 \quad$ Number of Liars After $\ell$ Deletions}

Lemma 5. After $\ell$ edge deletions in any graph $G$ of diameter $D$, every connected component of the resulting graph have diameter at most $D(\ell+1)$.

Proof. As claimed in [SBvL87], given $\ell$, the maximum diameter of the graph obtained by deleting $\ell$ edges from a graph $G$ of diameter $D$ is $D(\ell+1)$, assuming that the resulting graph is still connected. Now, if a single deletion disconnect in two parts a connected component of diameter $D$, both resulting components will have diameter at most $D$. So, after $\ell+1$ deletions, any connected component has at most diameter $D(\ell+1)$.

Theorem 4. Let $G$ be a n-nodes, m-edges graph of diameter $D$ without any liars. For any $\ell \leq m$, after $\ell$ edges deletion uniformly at random in $G$ the number of liars is $O\left(\frac{\ell^{2} D n}{m}\right)$.

Proof. As stated in Theorem 2, deleting one edge into a graph of diameter $D$ creates an average of at most $D(n-1) / m$ liars. From Lemma 5 , after the deletion of $\ell$ edges, the expected number of liars is

$$
\mathbb{E}(k) \leq \sum_{i=1}^{\ell} \frac{D i(n-1)}{m-(i-1)} \leq \sum_{i=1}^{\ell} \frac{D i(n-1)}{m-(\ell-1)} \leq \frac{D(n-1)}{m-(\ell-1)} \sum_{i=1}^{\ell} i
$$

or

$$
\mathbb{E}(k) \leq \frac{D(n-1)}{m-(\ell-1)} \times \frac{\ell(\ell-1)}{2}
$$




\section{Specific Topologies}

In this section, we show how tight the bounds are for some specific topologies. We just briefly describe the sketch of proofs. The study gives a justification for the introduction of the adversary model. In order to get tight bounds in the random fault model, we exhibit the worst configurations of advice and evaluate their probabilities in the random fault model.

Theorem 5. In the adversary model,

- $k=\Theta(n)$ for ErdsRnyi's random graphs with parameter $p=1 / 2$;

- $k=\Theta(\sqrt{n})$ for square grids;

- $k=\log _{2} n-1$ for hypercube.

In the random fault model,

- $k=\Theta(1 / n)$ for ErdsRnyi's random graphs with parameter $p=1 / 2$;

- $k=\Theta(1)$ for square grids;

- $k=\Theta(1 / \log n)$ for hypercube.

Here is some clue about the behaviour of the different graph families in the adversary model :

- ErdsRnyi's random graphs: each pair of nodes is connected with probability $p$. For $p=1 / 2$, almost all graphs have diameter 2 . If the deleted edge is between $L_{1}$ and $L_{2}$ then only 1 node can turn into a liar. However, a deletion between $L_{0}=\{t\}$ and $L_{1}$ can create $\Theta(n)$ liars since on average, there are $(n-1) / 4$ neighbours in $L_{2}$ of any individual node of $L_{1}$.

- grids: only nodes that share a coordinate (same row or column) with $t$ have $(s, t)$-arterial edges and thus can change their distance to $t$. The number of distance changes is then $|\mathcal{S}|=\Theta(\sqrt{n})$ for square grids. An adversary can force all neighbours of $\mathcal{S}$ to point to $\mathcal{S}$. From Lemma 2, we get that $k=\Theta(\sqrt{n})$.

- hypercube: only target's neighbours can increase their distance to $t$ after one edge deletion, so $|\mathcal{S}| \leq 1$ and only $k \leq \log _{2} n-1$ nodes of level $L_{2}$ can become liars.

In order to get tight bounds for the random fault model, we simulate the $m$ possible edge deletions and average $k$ :

- ErdsRnyi's random graphs: only edges leading to advice deletion can create liars. Condition on this event, on average, only $\Theta(1)$ liars appear. However, this event occurs with probability $\Theta(1 / n)$. In the other cases, no liar are obtained. 
- grids: with probability $1-\Theta(1 / \sqrt{n})$, there is no $(s, t)$-arterial edge between a random node and $t$. It follows that, with probability $1-\Theta(1 / \sqrt{n})$, we have at most one new liar (if the deleted edge contains an advice). With probability $\Theta(1 / \sqrt{n})$, we have $\Theta(\sqrt{n})$ liars.

- hypercube: only edges leading to an advice deletion or being neighbours of $t$ can create liars. However neighbours of $t$ can not become liars. For nodes of levels $L_{i \geq 2}$, there is no distance change after one edge deletion. Since $\mathbb{E}(\mathcal{F}(e))=\frac{n-1}{n \log _{2} n}=\Theta(1 / \log n)$, we have $\mathbb{E}(k)=O(1 / \log n)$. To get a lower bound of $\Omega(1 / \log n)$, we just have to consider the $n / 2$ closest nodes from $t$. The probability that the deleted edge is linked to one of these nodes is at least $1 / 2$ and condition on this event, with probability at least $\frac{1}{2 \log _{2} n}$, a new advice is required and create a liar.

\section{Conclusion}

This work shows the importance of the diameter for the number of distance changes and liars appearances in a dynamic graph model. Of course, it would be interesting to consider edge/node addition. Contrary to edge deletion, an edge addition can drastically change the distance within the graph. Even for grids, the number of distance changes would be $\Omega(n)$ after a random edge addition.

\section{References}

Ber09. Aaron Bernstein. Fully dynamic (2+ epsilon) approximate all-pairs shortest paths with fast query and close to linear update time. In FOCS, pages 693702. IEEE Computer Society, 2009.

BK09. Aaron Bernstein and David R. Karger. A nearly optimal oracle for avoiding failed vertices and edges. In Michael Mitzenmacher, editor, STOC, pages 101-110. ACM, 2009.

CG84. F. R. K. Chung and M. R. Garey. Diameter bounds for altered graphs. Journal of Graph Theory, 8(4):511-534, 1984.

CLPR10. Shiri Chechik, Michael Langberg, David Peleg, and Liam Roditty. fsensitivity distance oracles and routing schemes. In Proceedings of the 18th annual European conference on Algorithms: Part I, ESA'10, pages 84-96, Berlin, Heidelberg, 2010. Springer-Verlag.

DI04. Camil Demetrescu and Giuseppe F. Italiano. A new approach to dynamic all pairs shortest paths. J. ACM, 51(6):968-992, 2004.

DTCR08. Camil Demetrescu, Mikkel Thorup, Rezaul Alam Chowdhury, and Vijaya Ramachandran. Oracles for distances avoiding a failed node or link. SIAM J. Comput., 37:1299-1318, January 2008.

HIKN10. Nicolas Hanusse, David Ilcinkas, Adrian Kosowski, and Nicolas Nisse. Locating a Target with an Agent Guided by Unreliable Local Advice. In Proceedings of the 29th Annual ACM SIGACT-SIGOPS Symposium on Principles of Distributed Computing PODC 2010, pages 355-364, Zurich Suisse, 2010. ACM New York, NY, USA.

HKK04. N. Hanusse, E. Kranakis, and D. Krizanc. Searching with mobile agents in networks with liars. Discrete Applied Mathematics, 137:69-85, 2004. 
HKKK08. N. Hanusse, D. J. Kavvadias, E. Kranakis, and D. Krizanc. Memoryless search algorithms in a network with faulty advice. Theor. Comput. Sci., 402(2-3):190-198, 2008.

HS01. John Hershberger and Subhash Suri. Vickrey prices and shortest paths: What is an edge worth? In FOCS, pages 252-259, 2001.

KB10. Neelesh Khanna and Surender Baswana. Approximate shortest paths avoiding a failed vertex: Optimal size data structures for unweighted graphs. In Jean-Yves Marion and Thomas Schwentick, editors, STACS, volume 5 of LIPIcs, pages 513-524. Schloss Dagstuhl - Leibniz-Zentrum fuer Informatik, 2010.

Kin99. Valerie King. Fully dynamic algorithms for maintaining all-pairs shortest paths and transitive closure in digraphs. In FOCS, pages 81-91, 1999.

KK99. E. Kranakis and D. Krizanc. Searching with uncertainty. In Proc. SIROCCO'99, pages 194-203, 1999.

NPW03. Enrico Nardelli, Guido Proietti, and Peter Widmayer. Finding the most vital node of a shortest path. Theor. Comput. Sci., 296:167-177, March 2003.

SBvL87. A. Schoone, H. Bodlaender, and J. van Leeuwen. Improved diameter bounds for altered graphs. In Gottfried Tinhofer and Gunther Schmidt, editors, Graph-Theoretic Concepts in Computer Science, volume 246 of Lecture Notes in Computer Science, pages 227-236. Springer Berlin / Heidelberg, 1987.

Tho04. Mikkel Thorup. Fully-dynamic all-pairs shortest paths: Faster and allowing negative cycles. In Torben Hagerup and Jyrki Katajainen, editors, SWAT, volume 3111 of Lecture Notes in Computer Science, pages 384-396. Springer, 2004. 